\title{
Archivando la Web, el proyecto Padicat (Patrimonio Digital de Cataluña)
}

\author{
Por Ciro Llueca
}

Resumen: Desde 1996 varios paises han llevado a cabo estrategias para garantizar el acceso permanente a la producción digital propia: las páginas web y el resto de recursos digitales publicados en internet. Estas estrategias están orientadas a asegurar en la medida de las posibilidades tecnológicas actuales a la adecuación del ciclo documental clásico a las páginas web: la compilación, el procesamiento, la preservación y el acceso permanente a la producción digital. Las bibliotecas nacionales han sido a menudo impulsoras de estas acciones y en el caso español, la Biblioteca de

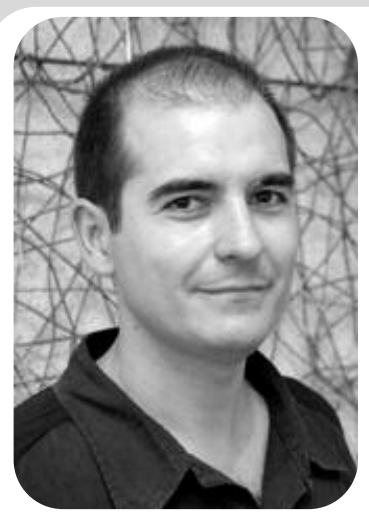
Ciro Llueca es diplomado en biblioteconomía y documentación por la Universitat de Barcelona, licenciado en documentación por la Universitat Oberta de Catalunya, y máster en documentación digital por la Universitat Pompeu Fabra. Es coordinador del proyecto Padicat (Patrimonio Digital de Cataluña) en la Biblioteca de Catalunya. Profesor asociado del Departament de Biblioteconomia y Documentació de la UB, profesor de los estudios de periodismo en la Universitat Abat Oliba CEU y consultor de los estudios de documentación de la UOC. Ha sido vicepresidente (2003-2006) del Col.legi Oficial de Bibliotecaris-Documentalistes de Catalunya. Catalunya $(B C)$ ha puesto en marcha el proyecto Padicat (Patrimonio Digital de Cataluña), dedicado al archivo de la web catalana. Este artículo presenta el proyecto Padicat.

Palabras clave: Repositorios digitales, Bibliotecas digitales, Archivos web, Bibliotecas nacionales.

\section{Title: Archiving the Web, the Padicat Project (Digital Heritage of Catalonia)}

Abstract: Since 1996 numerous countries have set into motion strategies for guaranteeing permanent access to their digital production: web pages and other digital resources published on the internet. The purpose of these strategies is to assure that the traditional documentary cycle can be adapted, to the extent that current technology permits, to web pages; allowing for the selection, processing, conservation and permanent availability of these digital resources. National libraries have often led these initiatives and, within Spain, the Biblioteca de Catalunya (BC) has initiated the Padicat project (Digital Heritage of Catalonia), devoted to archiving the contents of the Catalonian Web. This article describes the Padicat project.

Keywords: Digital repositories, Digital libraries, Web archives, National libraries.

Llueca, Ciro. "Archivando la Web, el proyecto Padicat (Patrimonio Digital de Cataluña)". En: El profesional de la información, 2006, noviembre-diciembre, v. 15, n. 6, pp. 473-478.

\section{El patrimonio digital}

Las tecnologías de la información y la comunicación han posibilitado que el patrimonio cultural y científico se presente en formato digital. Tal como expresa la Unesco en sus Directrices para la preservación del patrimonio digital ${ }^{1}$, los recursos que son fruto del conocimiento o la expresión de los seres humanos, ya sean de carácter cultural, educativo, científico o administrativo, o comprendan información técnica, jurídica, médica o de otro tipo, se generan cada vez más a menudo directamente en formato digi- tal, o se convierten a él a partir de material ya existente.

Desde 1996 las administraciones de varios países han llevado a cabo estrategias para garantizar el acceso permanente a la producción digital propia. Estas acciones están orientadas a asegurar, en la medida de las posibilidades tecnológicas actuales, la adecuación del ciclo documental clásico a las páginas web: la compilación, el procesamiento, la preservación y el acceso permanente a la producción bibliográfica digital.

El reto no es menor y las amenazas son múltiples: por una parte, la manifiesta obsolescencia del texto legal que posibilita el depósito legal en España. Complementariamente, el crecimiento exponencial de la producción digital, sumado a la baja permanencia de los materiales publicados en internet y, por supuesto y finalmente, el respeto a la legislación en materia de propiedad intelectual.

Pese a estas dificultades algunos países están realizando acciones de preservación de la producción digital más obvia: las webs. Las bibliotecas nacionales han sido muchas veces impulsoras de estas acciones, y en el caso español la $B i$ - 
blioteca de Catalunya (BC) puso en marcha en 2005 el proyecto Padicat (Patrimonio Digital de Catalu$\tilde{n} a)^{2}$, dedicado al archivo de la producción digital en Cataluña. El presente artículo detalla su funcionamiento ${ }^{3}$.

http://www.padicat.cat

\section{Depósitos digitales nacionales: proyectos existentes}

Un depósito (o repositorio) digital nacional es la herramienta fruto de la iniciativa dedicada a compilar, procesar y dar acceso a los recursos digitales de todo tipo creados en un territorio determinado, o sobre él. Estos "archivos web" son complementarios a los depósitos institucionales (como el E-Prints de la Universidad Complutense de Madrid o el DSpace Revistes y el DSpace Eprints de la Universitat Politècnica de Catalunya, entre muchas otras iniciativas); o temáticos (como la Biblioteca Virtual Miguel de Cervantes, el portal Tecnociencia e-revistas, o Temaria -que coordina la Facultat de Biblioteconomia $i$ Documentació de la Universitat de Barcelona-).

http://www.ucm.es/eprints

https://eprints.upc.es:8443/revistes https://eprints.upc.es:8443/dspace http://www.cervantesvirtual.com http://www.tecnociencia.es/e-revistas http://temaria.net

Existen diversos depósitos nacionales en funcionamiento, así como extensa bibliografía que los ha detallado y analizado ${ }^{4}$. Los más conocidos son también los que dieron los primeros pasos en 1996: el sueco Kulturarw3 y el australiano Pandora; así como un repositorio de alcance internacional, el gigante Internet Archive. Diez años más tarde podemos contar hasta 25 proyectos en diferentes fases de implementación, siendo acciones ya consolidadas un tercio de esta cifra.

\section{http://www.kb.se/kw3/ENG/}

http://pandora.nla.gov.au/index.html http://www.archive.org

El análisis de estas experiencias muestra dos modelos básicos de sistemas, con una tendencia generalizada hacia un modelo híbrido:

-el integral o exhaustivo (mayoritario, y característico especialmente de los países escandinavos), que persigue la integración automática de la web a partir de determinados criterios infraestructurales (lingüísticos, según el dominio de las
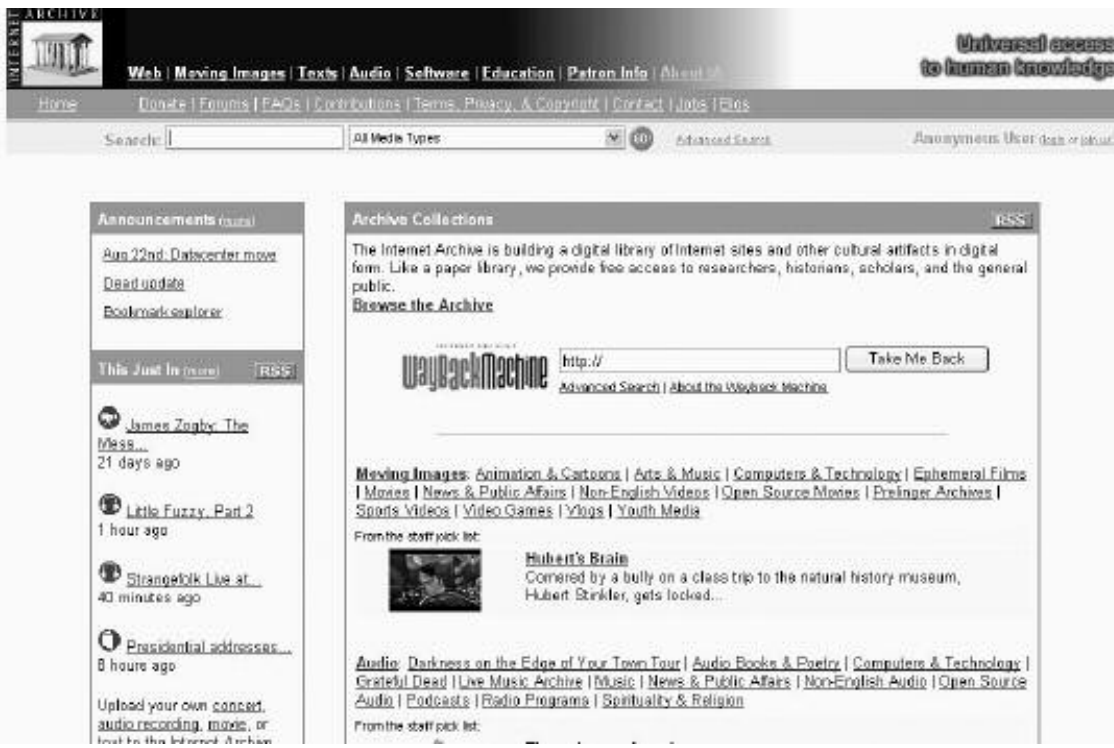

Figura 1. Internet Archive

páginas, la ubicación del servidor, etc.);

—el selectivo (asimilado por Australia, el Reino Unido o Japón, entre otros países), dirigido a compilar la web en base a escoger siguiendo criterios determinados (sobre un espacio geográfico, un tema de interés nacional, etc.).

Ambos han dado paso, en lo que es ya una tendencia generalizada, a modelos híbridos (cuyo caso más evidente es el de Dinamarca) que complementan la captura periódica de la web con acciones selectivas, y en ciertos casos ampliando además esa cobertura a acontecimientos concretos de interés social (elecciones, competiciones deportivas, etc.)

\section{La BC y el proyecto Padicat}

A las puertas de su centenario en 2007, la $B C$ ha apostado con firmeza por una serie de acciones para evolucionar hacia un modelo de biblioteca abierta, fiable y orientada al usuario. Una de las líneas estratégicas ha sido el impulso de proyectos digitales ${ }^{5}$, de carácter eminentemente cooperativo, para contribuir a la preservación del patrimonio catalán y aumentar la presencia de contenidos catalanes en internet. Algunos de esos proyectos son $A R$ $C A$ (Archivo de revistas catalanas antiguas), RACO (Revistas catalanas con acceso abierto), Claca (Clásicos catalanes) o el propio Padicat.

De hecho, tal como se expresa en las leyes catalanas de bibliotecas de 1981 y $1993^{6}$, la $B C$ tiene por misión recopilar, conservar y difundir la producción bibliográfica catalana y la relacionada con el ámbito lingüístico catalán, así como velar por la conservación y la difusión del patrimonio bibliográfico. Entendemos que este patrimonio bibliográfico incluye también la producción bibliográfica digital, objeto 
del proyecto que se presenta en este artículo.

Tomando la misión ya descrita, establecemos el objetivo genérico de Padicat: diseñar y producir un sistema que permita a la $B C$ compilar, procesar y dar acceso permanente a la producción digital catalana. Es un depósito digital que cuenta con la colaboración del Centre de Supercomputació de Catalunya (Cesca) y de la Secretaria de Telecomunicacions $i$ Societat de la Informació de la Generalitat de Catalunya.

En la línea de la ya referida tendencia generalizada en las bibliotecas nacionales, el modelo que adopta la $B C$ es el sistema híbrido, consistente en compilar masivamente las fuentes digitales publicadas en abierto en internet; impulsar los acuerdos selectivos con los agentes implicados en la producción digital en Cataluña; y promover líneas de investigación específicas por medio de la integración focalizada de recursos digitales sobre determinados acontecimientos de la vida pública catalana.

\section{Funcionamiento del sistema}

Se basa en el ciclo documental clásico de bibliotecas y servicios de información (compilación, proceso, difusión), y sin hacer un análisis detallado del sistema informático que lo permite, identificamos los pilares del proceso en la captura de los recursos, la organización de los mismos, y el acceso permanente a la colección.

En lo que respecta a la captura, se llevó a cabo un ejercicio de definición del tipo de recursos digitales susceptibles de captura, así como del alcance temático del proyecto. Es evidente que la tecnología que se aplica a los sistemas de repositorio digital cambia y cambiará en el futuro de forma importante; por ello las variables sobre la naturale-

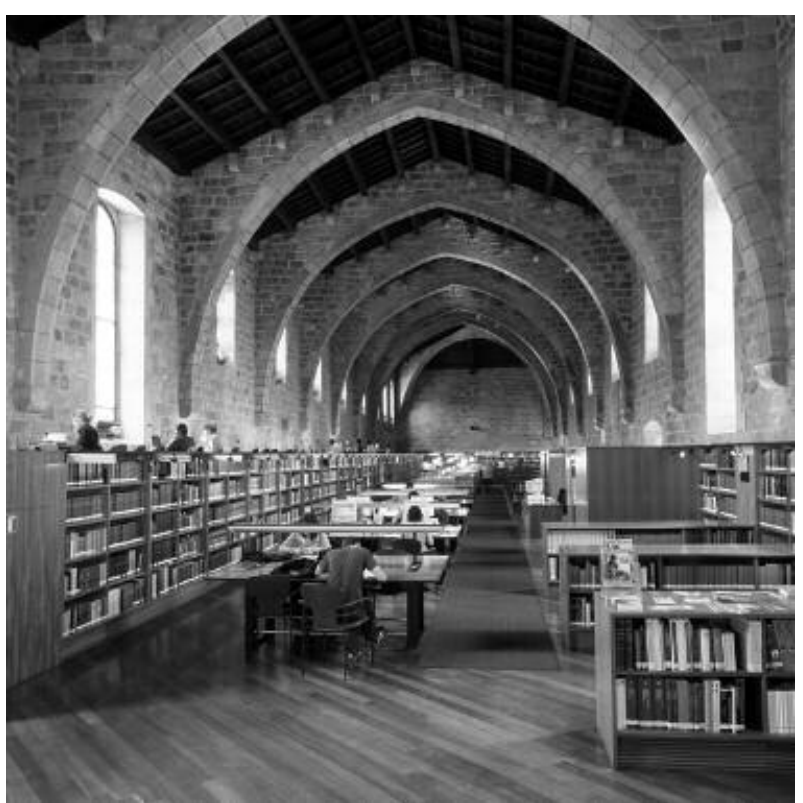

Figura 2. La Biblioteca de Catalunya

za del recurso digital y el software utilizado dotan de diferente grado de complejidad a lo que conocemos como páginas web.

Sin entrar a valorar el verdadero núcleo informativo de una web (¿toda ella?, ¿sólo la portada?, ¿algunos capítulos?, ¿un vídeo incrustado?), sí citaremos la definición usada habitualmente por los miembros del Laboratorio de Internet del Cindoc-CSIC, que servirá para definir qué entendemos genéricamente por recurso digital o web ${ }^{7}$ : una web es un conjunto de páginas ligadas jerárquicamente a una principal, identificables por una url y que forma una unidad documental reconocible e independiente de otras, bien por su temática, por su autoría o por su representatividad institucional.

Por tanto, entendemos que una web susceptible de formar parte de la colección del proyecto deberá cumplir dos condiciones básicas: será identificable por una url, y formará una unidad documental reconocible. No importa pues que ese recurso digital esté en un lenguaje de programación concreto, o que el formato sea texto, imagen, sonido, etc. De hecho, las primeras pruebas del proyecto arrojaron datos crista- linos sobre el elevado porcentaje de formatos estándares en las páginas web capturadas.

$$
\begin{gathered}
\text { “Padicat es un } \\
\text { depósito digital } \\
\text { pionero en } \\
\text { España } \\
\text { impulsado por la } \\
\text { Biblioteca de } \\
\text { Catalunya, con la } \\
\text { colaboración del } \\
\text { Centre de } \\
\text { Supercomputació } \\
\text { de Catalunya } \\
\text { (Cesca)” }
\end{gathered}
$$

Por lo que respecta a la cobertura temática del proyecto Patrimonio digital de Cataluña, entendemos:

-Patrimonio digital: como la información electrónica publicada en internet, en abierto o no, independientemente del formato en que se presenta.

-De Cataluña: en el sentido que tradicionalmente ha tenido la bibliografía nacional de Cataluña en que se basa la política de la $B C$ : 
todo aquello producido en, o que trate sobre Cataluña.

Lo cierto es que internet está diseñado para romper barreras políticas y hacer la información accesible universalmente. Pese a este hecho definitorio, es posible identificar partes de esa red que contengan módulos de interés de grupos concretos, a los que podemos llamar "comunidades de usuarios web", referidas a cierta temática o simplemente de interés de una comunidad concreta. A efectos prácticos, se establece la estrategia de captura de webs en: bajo dominio .cat, o ubicadas en servidores de Cataluña; bajo dominios geográficos o genéricos (.es, .com, .net, .org, etc.) en lengua catalana; o finalmente, aquellas que no cumplen los requisitos anteriores, pero están relacionadas temáticamente con Cataluña.

Tras la fase de análisis y testeo se decidió utilizar el programa informático Heritrix 8 , usado por la mayoría de los proyectos similares al que nos ocupa, para la captura.

La organización de los recursos web capturados debe permitir gestionar la colección y asegurar la recuperación, toda vez que ha de preservar los contenidos con las técnicas disponibles. Esta organización incluye la identificación permanente, la aplicación de metadatos, el almacenamiento y la preservación.

El programa $B A T^{9}$ fue el elegido para el procesamiento de los recursos digitales, pues forma parte de los módulos relacionados con Heritrix y el resto de software, siendo el que permite gestionar e indexar los archivos .arc, resultantes de la captura. En paralelo se estudia la incorporación de un sistema de gestión documental que permita la asignación de metadatos a una parte significativa de la colección, lo que garantizaría la posibilidad futura de integrarla en otros catálogos de la $B C \mathrm{u}$ otras instituciones.
Por otra parte, y como previsión, sería posible un depósito que permita conservar todos los recursos, de manera que se tenga acceso a ellos en todo momento. Se contempla también un sistema de doble copia en diferente ubicación geográfica, con una capacidad total de 20TB en los períodos de producción y explotación del proyecto, hasta 2008.

El método de preservación merece un tratamiento en un futuro artículo, pero no sorprenderemos a nuestros lectores si apuntamos que somos conscientes de la problemática de las estrategias más habituales de preservación ${ }^{10}$, como la migración periódica o refresh de los datos (migración a nuevas versiones de los mismos programas o lenguajes, o a nuevas aplicaciones capaces de leer los anteriores), la emulación (el uso de software, especificaciones, etc., utilizado en el momento de la creación), la recreación (simulación por ingeniería inversa u otros métodos).

En todo caso, las previsiones sobre el tipo de archivos que el proyecto deberá gestionar, basadas en los experimentos realizados, muestran que la mayor parte de los archivos corresponden a formatos estándar, que pueden simplificar la tarea preservadora al menos en las macrocifras. Así, en una de las pruebas realizadas para cubrir este detalle, obtenemos que de una muestra cercana a 3 millones de archivos, el $96 \%$ corresponde a formatos estándar: texto/html (1,6 millones, el 53\%), imagen jpeg o gif (0,9 millones, el 40\%), o pdf (75.000 archivos, que representan el $3 \%$ del total).

Para el acceso permanente a los recursos, una vez capturados e indexados, se apuesta por los módulos de búsqueda Nuth-Wax ${ }^{11}$ y de visualización Wera $^{12}$ y Wayback ${ }^{13}$.

\section{Aspectos legales}

Evidentemente, el texto legislativo de $1971^{14}$ que regula las adquisiciones de la $B C$ por depósito legal ignora la producción digital. En positivo, lo único que contempla es la tipología documental que afecta al objetivo del depósito, o sea la "misión esencial de recoger toda la producción bibliográfica nacional". De hecho, el objeto de la misión del depósito legal incluye la producción digital si ésta se considera bibliográfica, en el sentido biblioteconómico del concepto, que prescinde del soporte material que contenga la información.

Pero la realidad es más compleja: la situación de alegalidad de la producción digital en relación con el depósito legal ha facilitado que en todos los países del mundo -y a

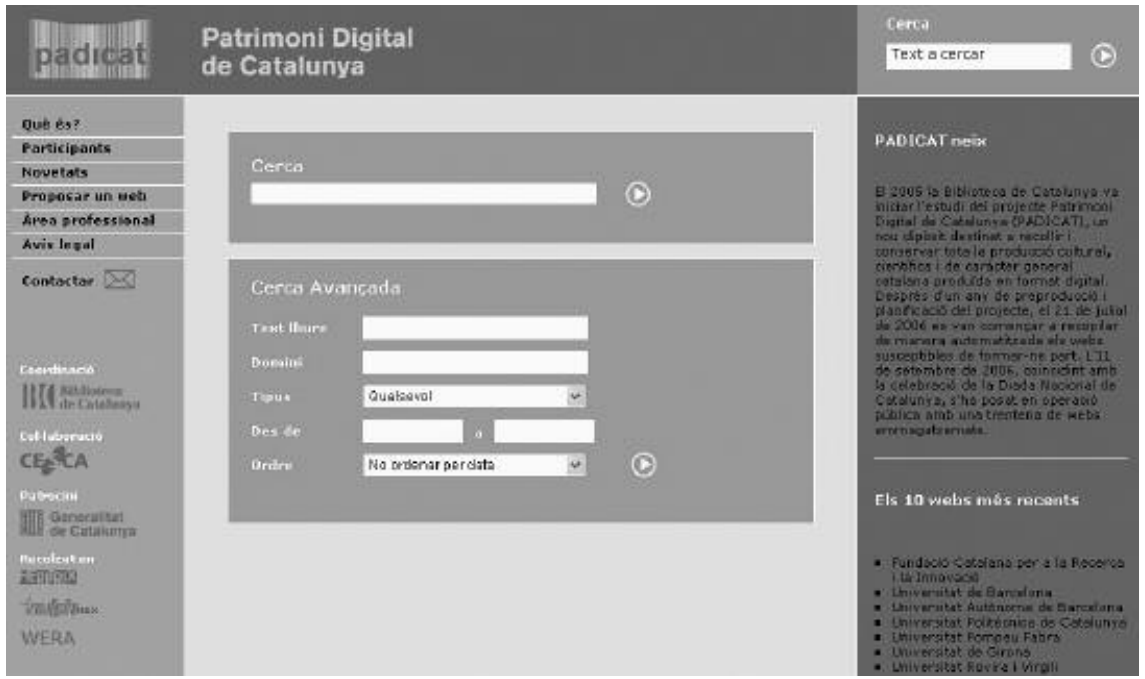

Figura 3. Web del proyecto Padicat 
semblanza del control bibliográfico de la producción impresa, realizado por las bibliotecas nacionales siglos más tarde que los primeros documentos impresos- no se haya producido un depósito sistemático de la producción digital por parte de los agentes productores que son, más que nunca, protagonistas de todo el ciclo editor (diseño, programación, redacción y publicación en un servidor).

“Presenta
indiscutibles
beneficios para
el sistema
bibliotecario y la
sociedad,
situando a la BC
en un escenario
privilegiado
como fuente de
información de la
producción
digital"

En todo caso, como indica Vives $(2005)^{15}$, disponemos de buenos y suficientes argumentos para convencer a nuestros depositantes de la bondad de los repositorios, sin entrar en debates estériles sobre la legalidad o no de preservar la producción digital. A título de ejemplo, ni en Suiza ni en los Países Bajos existen siquiera leyes de depósito legal ${ }^{16}$, y en todo caso los países actualmente preservadores están lejos de la legislación danesa ${ }^{17}$, que permite a su biblioteca nacional capturar cualquier web.

Más allá de la obsoleta regulación del depósito legal español, un referente que nos afecta por el hecho de compilar y sobre todo conservar y publicar en abierto, es la Ley de Propiedad Intelectual ${ }^{18}$, por la cual el autor "decidirá si su obra ha de ser divulgada y en qué forma" (art. 14.1).
Entenderemos, en primer lugar, que los recursos digitales son objeto de propiedad intelectual ${ }^{19}$. $\mathrm{Y}$ a partir del hecho de que las interpretaciones teóricas de la ley son diversas $^{20}$, asumimos que la localización, captura y copia de páginas web por parte de la $B C$ se ampara en el art. 37.1, puesto que nos hallamos ante un acto de investigación, sin finalidad lucrativa, y protegido por la tarea de conservación del patrimonio cultural que realiza la biblioteca. Una segunda fase posible, dedicada a transformar esa página web para asegurar su lectura futura, no debería observar problemas si esa página no contiene un programa informático que se transforma sin autorización expresa. Finalmente, y si tomamos la ley en su máxima literalidad, para dar acceso abierto en internet a la colección es preciso el consentimiento de los autores. No así para una comunicación en terminales offline, es decir, sin un acceso abierto en línea.

En respuesta a la literalidad de la ley, en todo caso, y con o sin cobertura legal, la voluntad de las entidades productoras (universidades y centros de investigación, administraciones públicas, empresas y gremios, colegios profesionales, asociaciones, partidos políticos, sindicatos, y particulares) han de observar Padicat como la oportunidad de ser los protagonistas de la futura investigación, así como de formar parte del patrimonio digital de Cataluña, entendido como un sistema útil para la sociedad y las instituciones que la forman. El ejemplo del Internet Archive, tras diez años de funcionamiento en abierto sin aparentes complicaciones legales, debe ser referencia obligada para las bibliotecas nacionales.

\section{Fases de implementación}

En junio de 2005 la $B C$ inició la fase de planificación, en la cual se realizó el análisis de los proyectos y recursos existentes, de los agentes implicados en la producción de webs en Cataluña, así como de los aspectos legales que condicionan las prácticas que se quieren llevar a cabo. Paralelamente se realizó una serie de pruebas de hardware y software que permitieron detectar a la $B C$ cuál es su capacidad en materia de captura y proceso de los datos. Finalmente, se documentó el escenario previsto de funcionamiento del sistema, así como los medios económicos, tecnológicos y de personal necesarios ${ }^{21}$.

El año 2006 ha sido la fase de producción, en la cual se está realizando el plan piloto del proyecto con la plena integración del socio tecnológico, el Cesca, al Padicat. En ese sentido, el objetivo es realizar varias acciones de captura exhaustiva de la Web catalana, así como llegar a los primeros acuerdos de colaboración con los agentes públicos y privados susceptibles de gestionar el depósito voluntario regular de sus páginas web. Adicionalmente se estudia la posibilidad de focalizar el proyecto en torno a un determinado acontecimiento de interés público.

Los años 2007 y 2008 se incluyen en la fase de explotación y creación de la oficina Padicat para realizar sistemáticamente las capturas y ampliar los acuerdos con los productores e, igualmente, las acciones dirigidas a gestionar focalizadamente determinados acontecimientos (sociales, culturales, políticos, etc.) susceptibles de crear futuras líneas de investigación.

2009 ha de permitir a la $B C$ y sus socios de proyecto contar con un escenario óptimo, en el cual funcione a pleno rendimiento este sistema, con unos indicadores cuantitativos de 100.000 páginas web capturadas en sus diversas ediciones. Son objetivos paralelos el cierre de acuerdos con 300 instituciones de todo tipo, así como permitir 
el acceso en abierto, en línea, a buena parte de la colección.

\section{Beneficios del proyecto}

Con el escenario descrito, los beneficios a ojos de los lectores de nuestro sector son evidentes: confección de la bibliografía nacional más allá de los formatos tradicionales, y posicionamiento de la $B C$ y los socios de proyecto en una situación privilegiada como fuente de información de cara al futuro.

Para el sistema bibliotecario, posibilidades infinitas de cooperación con el resto de instituciones de la memoria, sean bibliotecas, archivos o museos; impulso y liderazgo en la realización del patrimonio digital de España, a la espera de unir esfuerzos con el resto de bibliotecas responsables de la preservación del patrimonio digital del país. Finalmente, relación privilegiada con el resto de bibliotecas nacionales del mundo, en términos de preservación digital y depósitos web. Padicat representa indiscutibles beneficios para el sistema bibliotecario y la sociedad.

Para las instituciones, empresas, administraciones públicas y particulares que producen webs en Cataluña, preservación de la propia producción y garantía de acceso, con los condicionantes que la ley rige, a los contenidos y diseños que de otra manera probablemente desaparecerían.

Para la ciudadanía, y como se pretende en las directrices de la Unesco, acceso abierto y permanente a los recursos que son fruto del conocimiento y la expresión de los creadores del siglo XXI, sean de carácter cultural, educativo, científico o administrativo, o comprendan información técnica, jurídica, médica o de otro tipo.

\section{Notas}

1. Directrices para la preservación del patrimonio digital. Canberra: Unesco, 2003. Consultado en: 24-08-06. http://unesdoc.unesco.org/images/0013/001300/ 130071s.pdf

2. La web del proyecto Padicat está operativa desde septiembre de 2006

http://www.padicat.cat

3. El presente artículo revisa y complementa la comunicación: Llueca, Ciro. "El projecte Padicat (Patrimoni Digital de Catalunya) de la Biblioteca de Catalunya". En: 10es Jornades catalanes d'informació i documentació, 2006. Consultado en: 24-08-06.

http://eprints.rclis.org/archive/00006434/01/llue ca_padicat.pdf

4. Para una panorámica reciente véase: Llueca, Ciro. "Webs siempre accesibles: las bibliotecas nacionales y los depósitos digitales nacionales". En: BiD: textos universitaris de biblioteconomia i documentació, 2005, diciembre, n. 15. Consultado en: 24-08-06.

http://www2.ub.edu/bid/consulta_articulos.php? fichero $=15$ lluec $2 . \mathrm{htm}$

5. La estrategia y los proyectos han sido recientemente presentados en: Lamarca, Dolors; Serra, Eugènia. "L'estratègia de la Biblioteca de Catalunya en projectes digitals". En: Item, 2005, setembre-desembre, n. 41, pp. 41-43.

6. Artículo 7.1 de la "Llei de biblioteques de Catalunya, de 24 de abril de 1981". En: Diari oficial de la Generalitat de Catalunya, 1981, 29 abril, n. 123. Dicho artículo se refrendó en la "Llei 4/1993 del sistema bibliotecari de Catalunya, de 18 de marzo de 1993". En: Diari ofi cial de la Generalitat de Catalunya, 1993, 29 marzo, n. 1.727. Consultado en: 24-08-06. http://cultura.gencat.net/normativa/docs/LLEI_4 1993.doc

7. Interesante reflexión terminológica en: Pareja, Víctor Manuel [et al.]

8. Heritrix. Un artículo fundamental es: Mohr, Gordon [et al.]. "An introduction to Heritrix: an open source archival quality web crawler". En: International web archiving workshop, 2004. Consultado en: 24-08-06.

http://www.iwaw.net/04/proceedings.php?f=Mohr http://crawler.archive.org/

9. Bnf ArcTools.

http://crawlerarchive.org/cgi-bin/wiki.pl?back= BnfArcTools

10. Ayre, Catherine; Muir, Adrienne. "The right to preserve: the rights issues of digital preservation". En: D-lib magazine, 2004, March, v. 10, n. 3. Consultado en: 24-08-06.

http://www.dlib.org/dlib/march04/ayre/03ayre.html

11. NutchWax

http://archive-access.sourceforge.net/projects/ nutch/

12. Wera.

http://archive-access.sourceforge.net/projects/ weral

\section{Wayback.}

http://www.archive.org/web/web.php

14. "Orden de 30 de octubre de 1971, por la que se aprueba el Reglamento del Instituto Bibliográfico Hispánico". En: Boletín Oficial del Estado, 1971, 18 noviembre. En el capítulo II se regula el Depósito Legal. Fue cambiada parcialmente por la "Orden de 20 de febrero de 1973 del Ministerio de Educación y Ciencia por la que se modifican algunos artículos del Reglamento del Instituto Bibliográfico Hispánico". En: Boletín Oficial del Estado, 1973, 3 marzo.

15. Vives, Josep. "Aspectos de propiedad intelectual en la creación y gestión de repositorios institucionales". En: El profesional de la información, 2005, julio-agosto, v. 14, n. 4. Consultado en: 24-08-2006.

http://www.elprofesionaldelainformacion.com/ contenidos/2005/julio/267.pdf

16. Nos basamos en el exhaustivo artículo:

Pons, Amadeu. "El dipòsit legal dels recursos digitals: estat actual de la legislació en diversos països i projectes per endegar dipòsits nacionals de recursos digitals". En: Biblioteques digitals $i$ dipòsits nacionals de recursos digitals, 1999, pp. 25-54; y asimismo en la web de la biblioteca nacional australiana: National Library of Australia. Legal deposit. Consultado en: 24-08-06; así como en el sintético texto de: Cordón, José Antonio. "El Depósito legal y los recursos digitales en línea". En: Las bibliotecas nacionales del siglo XXI, 2005. Consultado en: 24-08-06.

http://www.nla.gov.au/padi/topics/67.html http://bv.gva.es/documentos/Ponencias/ Cordon.pdf

17. El texto: Act on legal deposit of published material: translation of Act No. 1439 of $22 \mathrm{De}$ cember 2004: unauthorized version. Recoge los últimos cambios a la ley danesa de 1998. Consultado en: 24-08-06.

http://www.bs.dk/content.aspx?itemguid $=\% 7 b 33$ 2484E6-A5B1-4CEE-B953-059843182050

18. "Real decreto legislativo 1/1996, de 12 de abril, por el que se aprueba el texto refundido de la Ley de propiedad intelectual, regularizando, aclarando y armonizando las disposiciones legales vigentes sobre la materia". En: Boletín oficial del Estado, 1996, 13 abril. Y la "Ley 23/2006, de 7 de julio, por la que se modifica el texto refundido de la Ley de propiedad intelectual...". En: Boletín oficial del Estado, 2006, 8 julio.

19. En el sentido de su artículo 10: "Son objeto de propiedad intelectual todas las creaciones originales literarias, artísticas o científicas expresadas por cualquier medio o soporte, tangible o intangible, conocido actualmente o que se invente en el futuro".

20. Dos textos rigurosos son: Vives, Josep. "Guia bàsica de propietat intel-lectual per a biblioteques". En: Item, 2004, n. 38. Consultado en: 24-08-06; y el ya citado en 15 .

http://www.raco.cat/index.php/Item/article/view/ 22629/39381

21. En el informe: Biblioteca de Catalunya. Memòria del plantjament del projecte Padicat (Patrimoni digital de Catalunya). Barcelona: Biblioteca de Catalunya, 2005. Consultado: 24-08-06.

http://www.bnc.es/bc/in_padicat_2005.pdf

Ciro Llueca, Coordinador Padicat (Patrimonio Digital de Cataluña), Biblioteca de Catalunya, Hospital, 56. 08001 Barcelona.

cllueca@bnc.cat 